Gut and Liver, Vol. 12, No. 1, January 2018, pp. 51-57

\title{
Characterizing Helicobacter pylori cagA in Myanmar
}

Thein Myint ${ }^{1}$, Muhammad Miftahussurur ${ }^{2,3,4}$, Ratha-korn Vilaichone ${ }^{5}, \mathrm{New} \mathrm{Ni}^{6}$, Than Than $\mathrm{Aye}^{7}$, Phawinee Subsomwong ${ }^{2}$, Tomohisa Uchida ${ }^{8}$, Varocha Mahachai ${ }^{9}$, and Yoshio Yamaoka ${ }^{2,3}$

${ }^{\prime}$ Department of Gastroenterology, Yangon General Hospital, University of Medicine (1), Yangon, Myanmar, ${ }^{2}$ Department of Environmental and Preventive Medicine, Oita University Faculty of Medicine, Yufu, Japan, ${ }^{3}$ Department of Medicine, Gastroenterology and Hepatology Section, Baylor College of Medicine, Houston, TX, USA, ${ }^{4}$ Gastroentero-Hepatology Division, Department of Internal Medicine, Dr. Soetomo Teaching Hospital-Faculty of Medicine-Institute of Tropical Disease, Universitas Airlangga, Surabaya, Indonesia, ${ }^{5}$ Gastroenterology Unit, Department of Medicine, Thammasat University Hospital, Pathumthani, Thailand, ${ }^{6}$ Department of Gastroenterology, Mandalay General Hospital, University of Medicine (Mandalay), Mandalay, Myanmar, ${ }^{7}$ Department of Gastroenterology, Thingangyun Sanpya General Hospital, University of Medicine (2), Thingangyun, Myanmar, ${ }^{8}$ Department of Molecular Pathology, Oita University Faculty of Medicine, Yufu, Japan, and ${ }^{9}$ GI and Liver Center, Bangkok Medical Center, Bangkok, Thailand

Background/Aims: Differences in the Helicobacter pylori infection rate are not sufficient to clarify the dissimilarity of gastric cancer incidence between Myanmar and its neighboring countries. To better understand this trend, the $\mathrm{H}$. pylori virulence gene cagA was characterized in Myanmar. Methods: Glutamate-proline-isoleucine-tyrosine-alanine (EPIYA) patterns and CagA multimerization (CM) motifs of cagA genotypes were examined by performing polymerase chain reactions and DNA sequencing. Results: Of 69 tested H. pylori strains, cagA-positive patients had significantly more severe histological scores in their antrum than cagA-negative patients. Sequence analysis revealed that $94.1 \%$ of strains had Western-type cagA containing an EPIYA motif (92.6\%) or EPIYT motif (6.4\%). The intestinal metaplasia scores in the antral of patients infected with the $A B C$ and $A B C C$ types of cagA were significantly higher than those of patients with $A B-$ type cagA. Interestingly, in patients infected with $H$. pylori, $46.3 \%$ of strains with three EPIYA motifs contained two identical Western-typical CM motifs, and these patients showed significantly higher antrum inflammation scores than patients infected with two identical nontypical-CM motif strains $(p=0.02)$. Conclusions: In Myanmarese strains, Western-type cagA was predominant. The presence of $\mathrm{CM}$ motifs and the proportion of multiple EPIYA-C segments might partially explain the intermediate gastric cancer risk found in Myanmar. (Gut Liver 2018;12:51-57)

Key Words: Helicobacter pylori; cagA; Myanmar

\section{INTRODUCTION}

Myanmar is the largest nation in mainland Southeast Asia with the gastric cancer age-standardized incidence rate (ASR) was reported to be 12.1 every 100,000 people per year based on GLOBOCAN 2012 (http://globocan.iarc.fr/), which is lower than China but much higher than Thailand or India (22.7, 3.1, and 6.1 per 100,000 people, respectively). However, the prevalence of Helicobacter pylori infection in Thailand (54.1\% to 76.1\%) $)^{1}$ and India $(58 \%)^{2}$ were even higher than that in Myanmar. In our previous study, we reported that the overall $H$. pylori infection rate in Myanmar was 48.0\% for patients (121/252) considered to be $H$. pylori-positive in no less than one out of five tests, including rapid urease test, culture, histology, immunohistochemistry, and serology. ${ }^{3}$ Therefore, the difference in the prevalence of $H$. pylori infection is not sufficient to elucidate the gastric cancer ASR differences between Myanmar and its neighboring countries.

Besides genetic and environmental components, the incidence of gastric cancer variety were reported to be clarified by $H$. pylori virulence factors' divergences, such as CagA, instead of the prevalence of $H$. pylori infection. ${ }^{4}$ Every CagA repeat region conceives glutamate-proline-isoleucine-tyrosine-alanine (EPIYA) motifs, denoted as EPIYA-A or EPIYA-B segments for the first repeat region, and EPIYA-C (Western-type cagA) or EPIYA$D$ (East Asian-type cagA) segments for the second repeat region. We previously showed that East Asian-type cagA was more virulent than Western-type cag $A,{ }^{5}$ partially due to a stronger binding affinity for Src homology 2 (SH2) containing protein-

\footnotetext{
Correspondence to: Yoshio Yamaoka

Department of Environmental and Preventive Medicine, Oita University Faculty of Medicine, 1-1 Idaigaoka, Hasama-machi, Yufu 879-5593, Japan Tel: +81-97-586-5740, Fax: +81-97-586-5749, E-mail: yyamaoka@oita-u.ac.jp

Received on February 1, 2017. Revised on April 21, 2017. Accepted on April 29, 2017. Published online October 27, 2017

pISSN 1976-2283 eISSN 2005-1212 https://doi.org/10.5009/gnl17053

Thein Myint and Muhammad Miftahussurur contributed equally to this work as first authors.

(a) This is an Open Access article distributed under the terms of the Creative Commons Attribution Non-Commercial License (http://creativecommons.org/licenses/by-nc/4.0) which permits unrestricted non-commercial use, distribution, and reproduction in any medium, provided the original work is properly cited.
} 
tyrosine phosphatase (SHP-2) and a greater ability to induce morphological changes in epithelial cells than Western-type cag $A .^{6-8}$ In addition, the number of " $C$ " sites among Westerntype cag $A$ varieties is directly correlated to the level of tyrosine phosphorylation and SHP-2-binding activity. ${ }^{9}$ We also previously reported that the number of "C" sites was associated with the severity of atrophic gastritis and gastric cancer in patients infected with Western-type cagA-positive strains. ${ }^{10,11}$

Among the EPIYA-B motif of Western-type CagA; EPIYA was the most common sequences, followed by EPIYT ${ }^{5,12,13}$ and it was associated with clinical outcomes. ${ }^{13,14}$ Based on the analysis of 364 Western-type CagA sequences, Zhang et al. ${ }^{13}$ revealed that gastric cancer cases were more belonged to the EPIYA sequence in the EPIYA-B motif rather than the EPIYT sequence, which might be due to differences in its affinity for phosphoinositol 3-kinase and induction of protein kinase B. In addition, we also confirmed that Indonesian patients that were infected with strains containing EPIYA sequences had less histological severity in their antrum than those with EPIYT sequences. This could predispose these patients to duodenal ulcer due to an opportunity of $H$. pylori to colonize in the duodenum and induce gastric metaplasia on the duodenal mucosa. ${ }^{14}$

Sixteen amino acids conserved motif sequence in the Cterminal variable region of CagA (e.g., FPLXRXXXVXDLSKVG), designated as CagA multimerization (CM) or CRPIA (conserved repeat responsible for phosphorylation-independent activity) motifs, is a prerequisite for the CagA-SHP-2 interaction, and it functions in $\mathrm{CM}$, playing a vital role in $H$. pylori pathogenesis. ${ }^{15,16}$ Among them, 11 amino acids are well conserved in both cagA genotypes. East Asian-type cag $A$ strains carries a single CM motif, located after the EPIYA-D segment, whilst two CM motifs placed within each and distal to the ending of EPIYA-C possesed by Western-type cagA strains. ${ }^{17}$ The interaction of $\mathrm{CM}$ and c-Met promotes inflammation and proliferation through nuclear factor $\kappa \mathrm{B}$ transcriptional activities and upregulation of $\beta$-catenin. ${ }^{18}$ The impact of CagA on host cells multimerization may influenced by the CM motifs frequency and variety, thus may influence the CagA competence to induce host cell disruption through SHP-2 deregulation. ${ }^{16}$

We hypothesized that characteristics of $H$. pylori cag $A$ in Myanmar are different than those in neighboring countries and become a determinant of gastric cancer incidence rate differences between these countries.

\section{MATERIALS AND METHODS}

\section{Patients and $H$. pylori}

We conducted a survey in both the largest cities of Myanmar, Yangon, and the second largest city, Mandalay. Gastric biopsy specimens for histological examination were embedded in paraffin after fixed into 10\% buffered formalin. Hematoxylin and eosin as well as May-Giemsa were selected as stain techniques.
The updated Sydney and the Operative Link on Gastritis Assessment (OLGA) system were used to analyze histological severity. ${ }^{19}$ Peptic ulcer and gastric cancer were identified by endoscopy, and gastric cancer was further confirmed by histopathology. Gastritis were determined based on any score of activity and inflammation (score $\geq 1$ ) in antral and/or corpus in histological examination. Informed consent agreement was sigantured by all participants, and the Ethics and Research Committee of the University of Medicine, Myanmar; Mandalay General Hospital; and Oita University Faculty of Medicine, Japan approved our protocol.

\section{H. pylori genotyping}

We cultured and isolated $H$. pylori colonies from biopsy specimens as previously described. ${ }^{20}$ The QIAamp DNA Mini Kit (QIAGEN, Valencia, CA, USA) was used to extract $H$. pylori DNA for $H$. pylori genotyping in accordance with the manufacturer's instructions. Polymerase chain reaction were used to determine the positivity of cagA and the bands were detected with electrophoresis (1.5\% agarose gel) in an ultraviolet transilluminator. ${ }^{5}$ The directs sequencing of cagA EPIYA repeat region and CM motif were performed using a Big Dye Terminator v3.1 Cycle Sequencing Kit (Applied Biosystems, Foster City, CA, USA) on a ABI 3130 Genetic Analyzer according to the manufacturer's instructions. Multiple sequence alignments of the cagA sequences were generated using MAFFT version 7 (available at http:// mafft.cbrc.jp/alignment/server/) and confirmed by visual inspection.

\section{Data analysis}

Data were analyzed using SPSS version 19.0 (IBM Corp., Armonk, NY, USA). Discrete variables were tested using the chisquare test; continuous variables were tested using the Mann-

Table 1. Histological Scores According to cagA Status

\begin{tabular}{lccc}
\hline & cagA (+) & cagA (-) & p-value \\
\hline Number & 61 & 8 & \\
Antrum & & & \\
$\quad$ Activity & $1.54(2)$ & $0.75(1)$ & 0.003 \\
$\quad$ Inflammation & $1.74(2)$ & $1.25(1)$ & 0.04 \\
Atrophy & $0.93(1)$ & $0.50(0.5)$ & 0.01 \\
Intestinal metaplasia & $0.16(0)$ & $0.00(0)$ & 0.32 \\
Corpus & & & \\
Activity & $0.87(1)$ & $0.75(1)$ & 0.69 \\
Inflammation & $1.10(1)$ & $1.25(1)$ & 0.25 \\
Atrophy & $0.28(0)$ & $0.5(0.5)$ & 0.13 \\
$\quad$ Intestinal metaplasia & $0.00(0)$ & $0.00(0)$ & 1.00 \\
OLGA score & $0.98(1)$ & $0.50(0.5)$ & 0.01 \\
\hline
\end{tabular}

Histological scores are presented as mean (median).

OLGA, Operative Link on Gastritis Assessment system. 
Whitney U test and t-test. A two-tailed p-value $<0.05$ was considered statistically significant.

\section{RESULTS}

We successfully isolated 69 Myanmarese strains (41 females and 28 males, mean age of $40.1 \pm 11.5$ years) from Yangon $(n=52)$ and Mandalay $(n=17)$ were examined by culture in our study. Sixty-five strains were isolated from gastritis patients, three were from duodenal ulcer patients, and one was from a gastric cancer patient. Patients from Mandalay contained slightly higher number of cagA-positive strains $(94.1 \%, 16 / 17)$ compared with those from Yangon $(86.5 \%, 45 / 52)(\mathrm{p}=0.40)$. Table 1 described the association of histological scores and cagA status. Scores for atrophy in the antrum were significantly lower in patients infected with cagA-negative strains than those with cagA-positive strains (mean [median], 0.50 [0.5] vs. 0.93 [1], $\mathrm{p}=0.01$ ). Activity and inflammation scores in the antrum of patients infected with cagA-positive strains were also significantly higher than in those with cagA-negative strains (mean [median], 1.54 [2] vs 0.75 [1], $\mathrm{p}=0.003 ; 1.74$ [2] vs 1.25 [1], $\mathrm{p}=0.04$, respectively). In contrast, these differences could not be observed in the corpus. None of the eight patients infected with cagAnegative strains had intestinal metaplasia in the antrum or corpus. The OLGA score in patients infected with cagA-positive strains was also significantly higher than in those infected with cagA-negative strains (mean [median], 0.98 [1] vs 0.50 [0.5], $\mathrm{p}=0.01)$.

\section{Characterization of the EPIYA motif in the cagA 3' repeat region}

Surprisingly, sequence analysis of the cagA 3' repeat region revealed that 41 strains were $A B C$ type, 10 were $A B C C$, two were $\mathrm{AB}$, one was $\mathrm{ABCCC}$, and one was ACC. All of these strains are classified as Western-type cagA (55/61, 90.2\%). In contrast, only five strains were $\mathrm{ABD}$ and one $\mathrm{ABBD}$, which are considered to be East Asian-type cagA (6/61, 9.8\%). Amino acid sequences phylogeny demonstrated that most of Western-type cagA was linked to the references from Asian countries (Fig. 1). There was no difference in cagA type between strains isolated from Yangon and Mandalay $(\mathrm{p}=0.38)$. Although there was no relationship between cagA type and clinical outcomes in the Myanmar population, all cases from peptic ulcer or gastric cancer patients were Western-type cagA, whilst all of East Asiantype cagA strains were isolated from patients with gastritis. There was no statistical difference in the histological scores between East Asian-type and Western-type cagA ( $>>0.05$ ), possible owing to the small sample size. However, the intestinal metaplasia score in the antrum of patients infected with $\mathrm{ABC}$ and ABCC-type strains were significantly higher than that of AB-type strains ( $p<0.0001$ and $p=0.02$, respectively).

Four types of EPIYA or EPIYA-like sequences were obtained

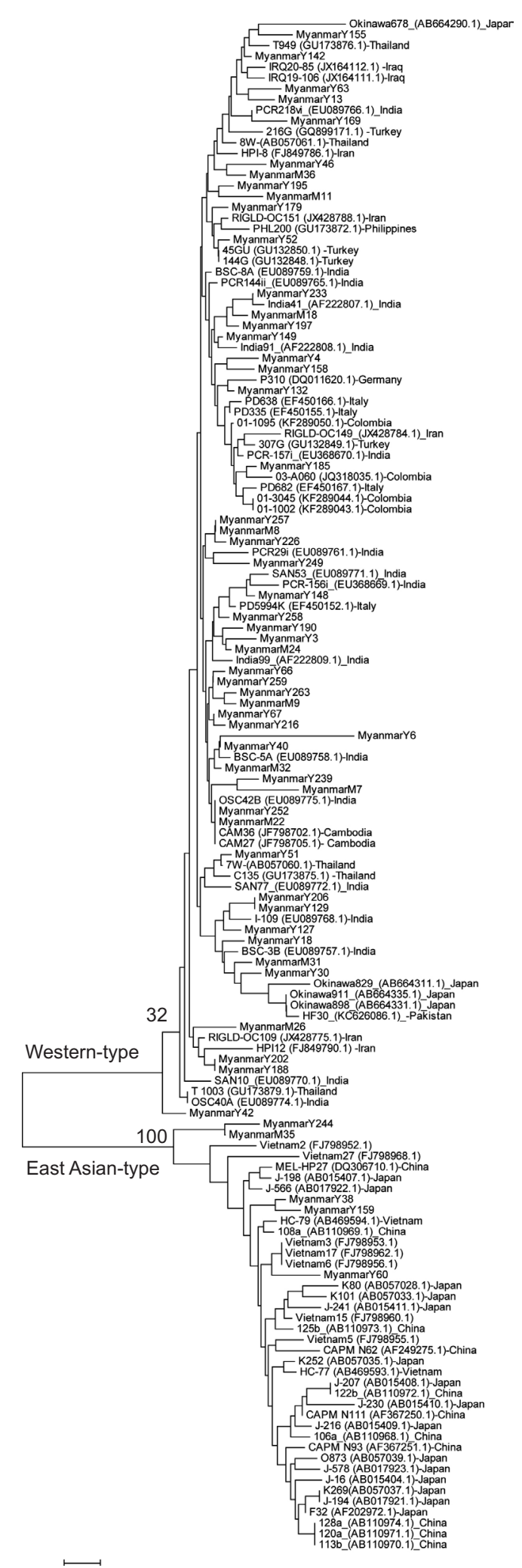

Fig. 1. Phylogeny analysis of cagA in Myanmarese strains. Phylogenetic trees were constructed using the neighbor-joining method, and estimated genetic distances were analyzed using the six-parameter method to compare Myanmarese strains against reference strains from GenBank. Main branch bootstrap values were calculated, and horizontal bar lengths represent the number of nucleotide substitutions per site. 
and the EPIYA motifs in each strain shown in Table 2. Among 61 CagA sequences, we found totally 189 EPIYA motifs. Thus, every CagA sequence contained approximately three EPIYA motifs in average. EPIYA was the most common among EPIYA motifs (175/189, 92.6\%), followed by EPIYT (12/189, 6.4\%). Segment B containing EPIYA was more predominant than EPIYT in Western-type cagA (78.3\% vs 20.0\%). There was no significant association between EPIYA motif type and histological score $(\mathrm{p}>0.05)$.

Table 2. Frequencies of EPIYA Motifs

\begin{tabular}{llrrrr}
\hline \multirow{1}{*}{ Type } & $\begin{array}{c}\text { All } \\
\text { motifs }\end{array}$ & Total & A motif B motif & $\begin{array}{c}\text { C or D } \\
\text { motif }\end{array}$ \\
\hline All cagA types & EPIYA & 175 & 61 & 47 & 67 \\
& EPIYT & 12 & 0 & 12 & 0 \\
& EPSYA & 1 & 0 & 0 & 1 \\
& ESIYG & 1 & 0 & 1 & 0 \\
& Total & 189 & 61 & 60 & 68 \\
Western-type cagA & EPIYA & 160 & 55 & 44 & 61 \\
& EPIYT & 10 & 0 & 10 & 0 \\
& EPSYA & 1 & 0 & 0 & 1 \\
& Total & 171 & 55 & 54 & 62 \\
East Asian-type cagA & EPIYA & 15 & 6 & 3 & 6 \\
& EPIYT & 2 & 0 & 2 & 0 \\
& ESIYG & 1 & 0 & 1 & 0 \\
& Total & 18 & 6 & 6 & 6 \\
\hline
\end{tabular}

EPIYA, glutamate-proline-isoleucine-tyrosine-alanine.

\section{Characterization of the $\mathrm{CM}$ motif}

Among 67 strains with cagA-positive, 25 contained the typical peptide sequence of Western countries strains (FPLKRHDKVDDLSKVG), placed within the EPIYA-C segment, immediately after and the last repeat. Only 19 of 41 strains (46.3\%) with three EPIYA motifs contained the Western CM twin motifs (Fig. 2). Interestingly, $24.4 \%$ of them possesed the nontypical twin CM motifs (FPLKKHDKVDDLSKVG), while 11 strains possesed a mix of typical Western and nontypical Western CM motifs (mixed-type CM motifs). Compared with the patients infected with strains containing typical Western CM motifs, patients with strains with nontypical Western CM motifs had significantly higher scores for antrum inflammation (mean [median], 2.15 [2] vs 1.56 [2], $p=0.02$ ). However, we found insignificant difference in histological scores between nontypical CM motifs and mixtype CM motifs ( $p>0.05)$.

\section{Nucleotide sequencing}

The cagA EPIYA repeat region and CM motif sequence data are registered with accession numbers LC209107 to LC209167 assigned by DDBJ.

\section{DISCUSSION}

Nearly all $H$. pylori isolates from East Asian countries and around three-quarter of isolates from Western countries are cagA positive and..$^{21,22}$ In Western countries, the peptic ulcer or gastric cancer risk prone to individuals infected with cagApositive $H$. pylori strains than those infected with cag $A$-negative strains. ${ }^{23}$ In this study, we found that cagA-positive patients had

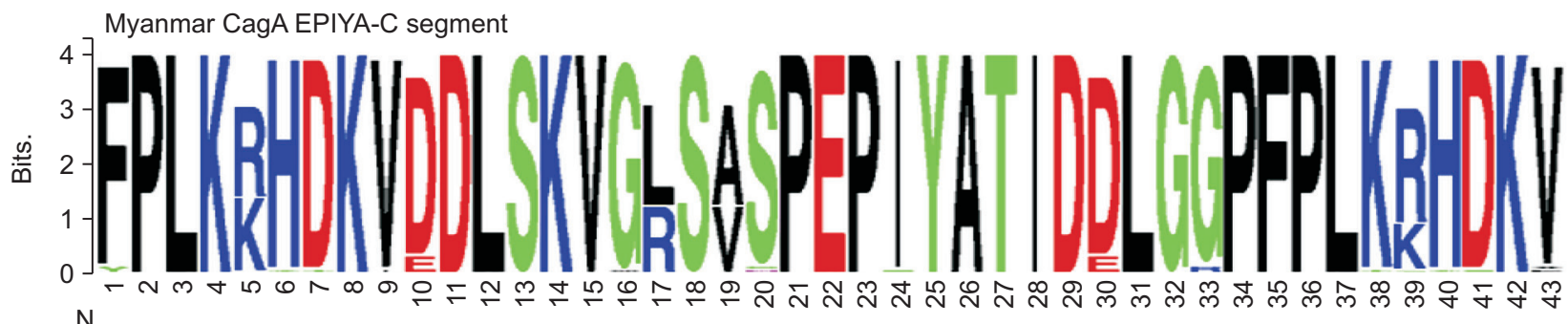

N

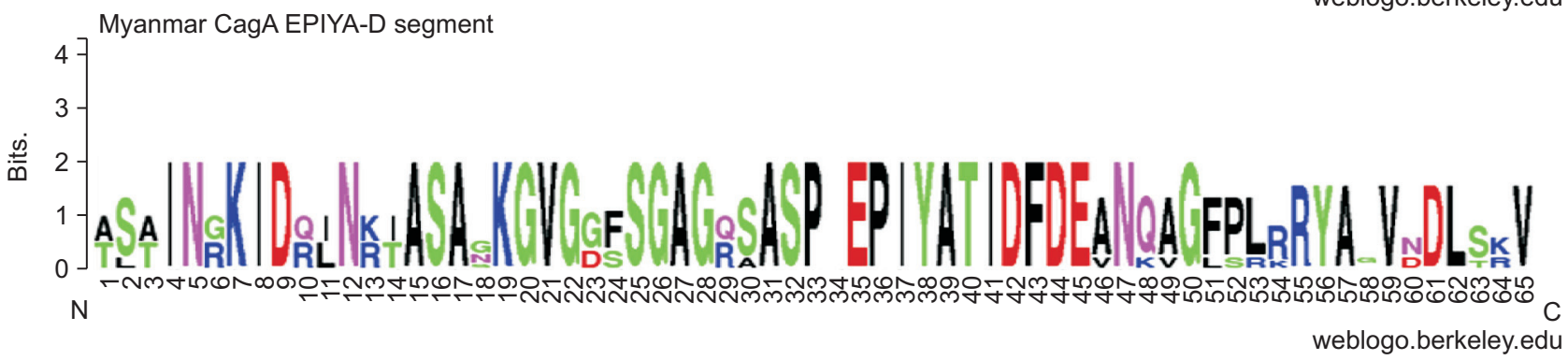

Fig. 2. CagA multimerization motifs in Western-type versus East Asian-type CagA from Myanmarese strains. WebLogo. v3 software (http://weblogo.threeplusone.com/) was used to analyze the sequence data. EPIYA, glutamate-proline-isoleucine-tyrosine-alanine. 
higher histological scores than cagA-negative patients. Moreover, none of gastric intestinal metaplasia observed in the eight cagA-negative patients suggesting the high-risk population in Myanmar are the patients infected with cagA-positive $H$. pylori strains as similar to Western countries.

In contrast with our prediction, most of the cagA sequences of the Myanmar strains were characterized as Western-type cagA (94.1\%), similar to that from the neighboring countries India and Pakistan. ${ }^{24,25}$ Myanmarese ancestry includes the TibetoBurman (Burmese-Lolo and Karen) branches of Sino-Tibetans that moved back southwards through Yunnan to Myanmar. These tribes began their migration from the north- and the east towards the Yangtze and Yellow River basins. ${ }^{26}$ Therefore, we previously believed that Myanmar would predominantly contain East Asian-type cagA or a minimally intermediate percentage of East Asian-type cagA, similar to Thailand. ${ }^{27}$ Considering the current population of Myanmar, $H$. pylori would also have been acquired from intermarriage of the various races and nationalities with indigenous ethnic groups, and the prevalence of East Asian-type cagA could have been reduced by competition with novel strains. However, unlike the usual South Asian population that has an "Asian paradox" phenomenon, suggesting that Western-type cagA is likely a marker of low virulence in these populations, Myanmar is a country characterized by an intermediate risk of gastric cancer. Moreover, the ASR rate of gastric cancer in Myanmar is higher than that of a neighboring country, Thailand, where approximately half are East Asian-type and half are Western-type strains. ${ }^{1,27}$ Interestingly, we also found that the proportion of strains with multiple EPIYA-C segments was higher in patients from Myanmar (11/55, 20.0\%) than in patients from Thailand $(10 / 75,13.3 \%)^{28}$ or India $(0 / 32,0 \%){ }^{29}$ In fact, patients infected with multiple EPIYA-C segments $H$. pylori had higher gastric cancer risk than in those infected with a single EPIYA-C segment strains, although only shown in Western countries. ${ }^{30,31}$ However, it is possible that the observation that strains with multiple EPIYA-C segments are less resistant to gastric acid ${ }^{11}$ may have resulted from a correlation; notably, the mean age of patients with multiple EPIYA-C segments in that study was lower than in those with single EPIYA-C segments. In this study, patients with $\mathrm{ABC}$ and $\mathrm{ABCC}$ types showed significantly higher intestinal metaplasia in the antrum than in those with the $\mathrm{AB}$ type. This data is also compatible with a previous study showing that $H$. pylori with single or multiple EPIYA-C motifs is associated with a higher risk of gastric cancer than $H$. pylori with only $\mathrm{AB}$ motifs. ${ }^{32,33}$ However, the lack of significant histological difference between single and multiple EPIYA-C might simply be due to the small number of multiple EPIYA-C strains. Although we revealed that among Western-type cagA, EPIYA sequence in the EPIYA-B motif was the predominant with only a low proportion of EPIYT, similar to that found for India ${ }^{29}$ thus, it is not a determinant to explain the differing risk of gastric cancer between Myanmar and India.
Several studies have reported important proinflammatory and pro-oncogenic activities of CagA that are independent of the EPIYA motifs. ${ }^{16,17}$ Sicinschi et al. ${ }^{17}$ reported that specific Western/Western CM motif strains have more potent affinity between CagA and SHP-2 compared to that of Western/East Asian CM motif strains, suggesting that this may be as important for disease incidence. They also found that subjects from low gastric cancer risk areas were infected with Western/East Asian CM motif strains. Interestingly, in our study, almost half of strains contained a nontypical CM motif, which are associated with higher inflammation than a specific Western motif. Our unpublished data showed almost all CM motifs in Thailand have another specific Western motif. Therefore, the types of CM motifs might partly clarify the distinction in gastric cancer risk amongst Myanmar and Thailand.

Nevertheless, we also should take into account that the genetic and environmental factors may involve on gastric cancer risk determination in addition to influence of virulence factors towards gastric carcinogenesis. For example, the northern region of Thailand has the highest incidence rate of gastric cancer; in contrast, the southern region has the lowest rates. ${ }^{34}$ This difference may possibly be due to consumption of foods high in nitrates and consumption of vegetables low in ascorbic acid, which was significantly higher in the northern and northeast regions than in the southern region. In Malaysia, the rate of gastric cancer was five times lower among Malay than that in Chinese or Indians. ${ }^{35}$ Myanmar is subdivided into more than 100 ethnic groups; among them, the Bamar represent 68\% of the population. Other common minorities are the Shan (10\%), Karen (7\%), Arakanese (4\%), Chinese (3\%), and the ethnolinguistically related Mon and Khmer (2\%). These ethnic groups have diverse linguistic and cultural subgroups, religions, and different geographic locations. We isolated $H$. pylori from the two biggest cities in Myanmar, Yangon and Mandalay. In both of those cities, the Bamar are the major ethnic group present. Several ethnic groups have different ancestors than the Bamar, which might be important in distinguishing gastric cancer risk in the population of Myanmar. For example, the Karen ethnic group has a different genetic composition than the Bamar by mitochondrial DNA and also CYP2C19*3 allele analysis. ${ }^{26,36}$ On the other hand, Myanmar has a lower consumption of fruits and vegetables (0.19 vs 0.35 and $0.23 \mathrm{~kg} /$ day/capita) but lower consumption of alcohol and tobacco (0.0006 vs 0.0229 and $0.0025 \mathrm{~L}$ of alcohol/day/ adult and 0.4 vs 2.9 and 3.8 cigarettes/day/adult, respectively) than Thailand and India, respectively. ${ }^{37}$ Overall, the genetic and environmental components may be more relevant than bacterial factors for considering gastric cancer risk in Myanmar. Further studies are necessary to better elucidate the contribution of host and environmental factors for the different gastric cancer risk between Myanmar and Thailand.

The small number of strains obtained in this study was one of its primary limitations. Further studies with increased sample 
numbers, balanced for each diagnosis, and including ethnogeographic region are prominent to better elucidate the influence of $H$. pylori virulence genes towards clinical outcomes.

In conclusion, to our knowledge, this is the first study that elucidated the diverse characteristics of the cagA region of $H$. pylori strains in Myanmar. We found that most Myanmarese strains had a Western-type cagA genotype. The CM motifs and proportion of multiple EPIYA-C segments, which may partly explain differences in the gastric cancer rate between Myanmar and Thailand or India. However, it is possible that host genetic and environment factors may be more responsible for gastric cancer risk in Myanmar than bacterial factors.

\section{CONFLICTS OF INTEREST}

No potential conflict of interest relevant to this article was reported.

\section{ACKNOWLEDGEMENTS}

This study was funded by grants from the National Institutes of Health (DK62813) and the Grants-in-Aid for Scientific Research from the Ministry of Education, Culture, Sports, Science, and Technology (MEXT) of Japan (25293104, 26640114, 15H02657, and 221S0002) (Y.Y.). It was also supported by the Japan Society for the Promotion of Science (JSPS) Institutional Program for Young Researcher Overseas Visits (Y.Y.) and the Strategic Funds for the Promotion of Science and Technology from Japan Science and Technology Agency (JST; Y.Y.). All authors have read and approved the final version of the manuscript.

Author contributions: T.M., R.V., Y.Y., and V.M. designed the study; T.M., R.V., N.N., T.T.A., V.M., T.U., and Y.Y. provided the collection of samples; M.M. and P.S. performed data analysis and interpretation; M.M. and Y.Y. analyzed and interpreted data and wrote the manuscript, Y.Y. revised the manuscript to include important content. All authors read and approved the final version of the manuscript.

\section{REFERENCES}

1. Sahara S, Sugimoto M, Vilaichone RK, et al. Role of Helicobacter pylori cagA EPIYA motif and vacA genotypes for the development of gastrointestinal diseases in Southeast Asian countries: a metaanalysis. BMC Infect Dis 2012;12:223.

2. Sodhi JS, Javid G, Zargar SA, et al. Prevalence of Helicobacter pylori infection and the effect of its eradication on symptoms of functional dyspepsia in Kashmir, India. J Gastroenterol Hepatol 2013;28:808-813.

3. Myint T, Shiota S, Vilaichone RK, et al. Prevalence of Helicobacter pylori infection and atrophic gastritis in patients with dyspeptic symptoms in Myanmar. World J Gastroenterol 2015;21:629-636.
4. Yamaoka Y. Mechanisms of disease: Helicobacter pylori virulence factors. Nat Rev Gastroenterol Hepatol 2010;7:629-641.

5. Matsunari 0, Shiota S, Suzuki R, et al. Association between Helicobacter pylori virulence factors and gastroduodenal diseases in Okinawa, Japan. J Clin Microbiol 2012;50:876-883.

6. Backert S, Moese S, Selbach M, Brinkmann V, Meyer TF. Phosphorylation of tyrosine 972 of the Helicobacter pylori CagA protein is essential for induction of a scattering phenotype in gastric epithelial cells. Mol Microbiol 2001;42:631-644.

7. Higashi H, Tsutsumi R, Fujita A, et al. Biological activity of the Helicobacter pylori virulence factor CagA is determined by variation in the tyrosine phosphorylation sites. Proc Natl Acad Sci U S A 2002;99:14428-14433.

8. Selbach M, Moese S, Hauck CR, Meyer TF, Backert S. Src is the kinase of the Helicobacter pylori CagA protein in vitro and in vivo. J Biol Chem 2002;277:6775-6778.

9. Higashi H, Tsutsumi R, Muto S, et al. SHP-2 tyrosine phosphatase as an intracellular target of Helicobacter pylori CagA protein. Science 2002;295:683-686.

10. Yamaoka Y, Kodama T, Kashima K, Graham DY, Sepulveda AR. Variants of the 3' region of the cagA gene in Helicobacter pylori isolates from patients with different $\mathrm{H}$. pylori-associated diseases. J Clin Microbiol 1998;36:2258-2263.

11. Yamaoka Y, El-Zimaity HM, Gutierrez 0, et al. Relationship between the cagA 3' repeat region of Helicobacter pylori, gastric histology, and susceptibility to low pH. Gastroenterology 1999;117:342-349.

12. Xia Y, Yamaoka Y, Zhu Q, Matha I, Gao X. A comprehensive sequence and disease correlation analyses for the C-terminal region of CagA protein of Helicobacter pylori. PLoS One 2009;4:e7736.

13. Zhang XS, Tegtmeyer N, Traube L, et al. A specific A/T polymorphism in Western tyrosine phosphorylation B-motifs regulates Helicobacter pylori CagA epithelial cell interactions. PLoS Pathog 2015;11:e1004621.

14. Miftahussurur M, Syam AF, Makmun D, et al. Helicobacter pylori virulence genes in the five largest islands of Indonesia. Gut Pathog 2015;7:26.

15. El-Etr SH, Mueller A, Tompkins LS, Falkow S, Merrell DS. Phosphorylation-independent effects of CagA during interaction between Helicobacter pylori and T84 polarized monolayers. J Infect Dis 2004;190:1516-1523.

16. Ren S, Higashi H, Lu H, Azuma T, Hatakeyama M. Structural basis and functional consequence of Helicobacter pylori CagA multimerization in cells. J Biol Chem 2006;281:32344-32352.

17. Sicinschi LA, Correa P, Peek RM, et al. CagA C-terminal variations in Helicobacter pylori strains from Colombian patients with gastric precancerous lesions. Clin Microbiol Infect 2010;16:369-378.

18. Suzuki M, Mimuro H, Kiga K, et al. Helicobacter pylori CagA phosphorylation-independent function in epithelial proliferation and inflammation. Cell Host Microbe 2009;5:23-34.

19. Dixon MF, Genta RM, Yardley JH, Correa P. Classification and grading of gastritis: the updated Sydney system. International 
Workshop on the Histopathology of Gastritis, Houston 1994. Am J Surg Pathol 1996;20:1161-1181.

20. Yamaoka Y, Kodama T, Kita M, Imanishi J, Kashima K, Graham DY. Relationship of vacA genotypes of Helicobacter pylori to cagA status, cytotoxin production, and clinical outcome. Helicobacter 1998;3:241-253.

21. Suzuki R, Shiota S, Yamaoka Y. Molecular epidemiology, population genetics, and pathogenic role of Helicobacter pylori. Infect Genet Evol 2012;12:203-213.

22. Yamaoka Y, Kodama T, Gutierrez O, Kim JG, Kashima K, Graham DY. Relationship between Helicobacter pylori iceA, cagA, and vacA status and clinical outcome: studies in four different countries. J Clin Microbiol 1999;37:2274-2279.

23. van Doorn LJ, Figueiredo C, Sanna R, et al. Clinical relevance of the cagA, vacA, and iceA status of Helicobacter pylori. Gastroenterology 1998;115:58-66.

24. Mukhopadhyay AK, Kersulyte D, Jeong JY, et al. Distinctiveness of genotypes of Helicobacter pylori in Calcutta, India. J Bacteriol 2000;182:3219-3227.

25. Yamaoka Y, Orito E, Mizokami M, et al. Helicobacter pylori in North and South America before Columbus. FEBS Lett 2002;517:180-184.

26. Summerer M, Horst J, Erhart G, et al. Large-scale mitochondrial DNA analysis in Southeast Asia reveals evolutionary effects of cultural isolation in the multi-ethnic population of Myanmar. BMC Evol Biol 2014;14:17.

27. Vilaichone RK, Mahachai V, Tumwasorn S, Wu JY, Graham DY, Yamaoka Y. Molecular epidemiology and outcome of Helicobacter pylori infection in Thailand: a cultural cross roads. Helicobacter 2004;9:453-459.

28. Chomvarin C, Phusri K, Sawadpanich K, et al. Prevalence of cagA EPIYA motifs in Helicobacter pylori among dyspeptic patients in northeast Thailand. Southeast Asian J Trop Med Public Health
2012;43:105-115.

29. Tiwari SK, Sharma V, Sharma VK, et al. Phylogenetic analysis, based on EPIYA repeats in the cagA gene of Indian Helicobacter pylori, and the implications of sequence variation in tyrosine phosphorylation motifs on determining the clinical outcome. Genet Mol Biol 2011;34:280-285.

30. Argent RH, Kidd M, Owen RJ, Thomas RJ, Limb MC, Atherton JC. Determinants and consequences of different levels of CagA phosphorylation for clinical isolates of Helicobacter pylori. Gastroenterology 2004;127:514-523.

31. Azuma T, Yamakawa A, Yamazaki S, et al. Correlation between variation of the 3' region of the cagA gene in Helicobacter pylori and disease outcome in Japan. J Infect Dis 2002;186:1621-1630.

32. Basso D, Zambon CF, Letley DP, et al. Clinical relevance of Helicobacter pylori cagA and vacA gene polymorphisms. Gastroenterology 2008;135:91-99.

33. Batista SA, Rocha GA, Rocha AM, et al. Higher number of Helicobacter pylori CagA EPIYA C phosphorylation sites increases the risk of gastric cancer, but not duodenal ulcer. BMC Microbiol 2011;11:61.

34. Mitacek EJ, Brunnemann KD, Suttajit M, et al. Geographic distribution of liver and stomach cancers in Thailand in relation to estimated dietary intake of nitrate, nitrite, and nitrosodimethylamine. Nutr Cancer 2008;60:196-203.

35. Lim KG. Malays in peninsular Malaysia may have the lowest incidence of stomach cancer in the world. Med J Malaysia 2009;64:91-92.

36. Tassaneeyakul W, Mahatthanatrakul W, Niwatananun K, et al. CYP2C19 genetic polymorphism in Thai, Burmese and Karen populations. Drug Metab Pharmacokinet 2006;21:286-290.

37. Lunet N, Barros H. Helicobacter pylori infection and gastric cancer: facing the enigmas. Int J Cancer 2003;106:953-960. 